\title{
Menopause for thought
}

\section{Linda Partridge}

IN the twilight of their careers eminent scientists not infrequently enter a philosopause, in which academic activity shifts gradually from the laboratory bench to the armchair. The menopause of the human female is both much more abrupt and much harder to understand. Simply put, natural selection is expected to cause the evolution of life histories that maximize the number of surviving progeny left by individuals. Why then does the fertility of women cease totally at a time of life when life expectancy is still high, thereby seeming to reduce lifetime reproductive success? In a paper in Evolutionary Ecology ${ }^{1}$, Alan Rogers has revisited this question, bringing an age-structured theory of kin selection to bear on it.

Cessation of fertility at the menopause is a consequence of failure of the ovaries to produce the egg-bearing follicles. Follicle number declines and they become less responsive to gonadotrophic hormones. Hormonal changes such as the drop in oestrogen level are a secondary consequence of this primary reproductive failure. The unique feature of the human menopause is the specific shut-down of fertility at a time when other physiological systems continue to function.

One explanation of the menopause is that it is an artefact of the high survival rates in modern human societies; it would not have been seen in the harsher circumstances under which the human life history evolved, because death would have already intervened. However, demographic data do not support this idea $^{2}$. Furthermore, it explains neither why a menopause is absent in other mammals, including chimps and gorillas, when survival rates are increased in benign conditions, nor why it is absent in human males.

The idea that the menopause could have evolved by natural selection was explored informally by G. C. Williams ${ }^{3}$. In pre-agricultural societies, death in childbirth would have been common, and its probability would have increased with the age of the mother. Human juveniles are also dependent on their mother for their survival for several years after birth, and her ability to care for them would decline with her age. Under these circumstances, it might pay a woman of 45 or 50 to devote the whole of her declining energies to the care of her dependent children and grandchildren, and to avoid putting them at risk of losing her through the increasing hazards of childbirth. Her loss of fertility would therefore be compensated by the increased survival or fertility of her existing descendants, and the menopause would evolve as a natural contraceptive. An alternative method would have been behavioural avoidance of mating but, given the predilections of males, a physiological method may have been safer.

Arguments such as these, despite their intuitive appeal, can be a misleading guide to the course of evolution. What Rogers has done is to formalize Williams's thinking, and make explicit the circumstances under which a new, rare allele causing continued fertility at the normal age of menopause would invade the population. The model allows the allele to have effects on the fertility and survival both of the mother and of her descendants, and extends existing models of kin selection to include effects at different ages and also the occurrence of time-delays between the cost of the act of altruism (maternal sterility with onset at menopause) and the benefit to the recipient (increased survival or fertility of descendants).

The first type of allele considered was one that caused continued fertility at the usual age of menopause, at the cost of an increasing risk of maternal death during childbirth. If maternal death occurred, then the survival and fertility of existing dependants was impaired. The second allele involved a different potential cost of continuing fertility, namely reduced ability to care for existing children during pregnancy and immediately after childbirth, exacerbated by the increasing age of the mother

Using data from the 1906 population of Taiwan, an agricultural society for which good demographic data are available, Rogers evaluated the action of natural selection on alleles of these two kinds. Female fertility declined markedly with age before the menopause in this population, and it followed that most children of women who reached menopause were already of reproductive age, while grandchildren were still pre-reproductive. Care was therefore likely to benefit mainly the fertility of offspring and the survival of grandchildren.

Rogers shows that mortality in childbirth increasing with maternal age cannot account for the evolutionary stability of the menopause. Using an estimate of probability of death during childbirth of 1 in a 100 , and assuming that maternal care doubled the fertility of children and resulted in 100 per cent survival of children and grandchildren until they were 10 , menopause was still strongly selected against. These assumptions were all stacked towards finding a benefit for the menopause, and even a tenfold increase in maternal mortality in childbirth with increasing age left the conclusion un- altered, so it seems robust. The peculiar hazards of human birth therefore seem unlikely to account for the existence of the menopause.

Next, Rogers considered the adverse effects of continuing fertility on care of existing dependants. Again stacking the assumptions in favour of menopause, and in particular assuming that adverse effects on maternal care of other descendants lasted until a new child was three years old, menopause became marginally evolutionarily stable. But with more realistic assumptions it seems likely that the benefit would be reversed. At first sight, therefore, the evolution of menopause by natural selection seems implausible.

An advantage of a theoretical model is that it makes assumptions explicit, and encourages measurement of real values of the important variables. What we notably lack for models of the evolution of the menopause is any direct information on what would happen to maternal survival and fertility if it did not occur. Several factors were not considered in Rogers's model, as he himself points out.

Increases in maternal mortality during birth and in the loss of care to dependent offspring because of the arrival of a new child may not be the only considerations, and a number of other processes could favour the menopause. The likelihood of death of women increases after the menopause because of ageing, in the absence of birth of new children. If women remained fertile at menopause, then each new child would anyway become progressively less likely to survive because of increasing likelihood of maternal death at times other than during childbirth. Continuing fertility might also cause a more rapid acceleration of maternal mortality rates. Furthermore, fertility has already declined at the time menopause occurs, so that its abolition is less costly then than it would be at a younger age.

Finally, pregnancy and very young children are more physically demanding for mothers than are older children. It seems probable that the main adverse effect of increasing maternal age on parental care would be on fetuses and very young children rather than on older ones, again tilting the evolutionary odds towards menopause. During their evolution other mammals and human males may not have displayed the protracted, costly and widely dispensed care of descendants seen in human females, and it may be this difference that accounts for the unique occurrence of the menopause.

Linda Partridge is in the Institute of Cell, Animal and Population Biology, University of Edinburgh, Ashworth Building, West Mains Road, Edinburgh EH9 3JT, UK.

Williams, G. C. Evolution 11, 398-411(1957)
} 